\title{
Application of Posture Recognition Service System Based on Information Fusion Smart Sensor in Dance Training
}

\author{
Yan Gao ${ }^{1,2}$ and Dazhi Xu iD $^{3,4}$ \\ ${ }^{1}$ College of Art, Hunan University of Arts and Science, Changde, 415000 Hunan, China \\ ${ }^{2}$ School of Management, Shinawatra University, Bangkok 12160, Thailand \\ ${ }^{3}$ College of Economics and Management, Hunan University of Arts and Science, Changde, 415000 Hunan, China \\ ${ }^{4}$ School of Public Administration, Central South University, Changsha, 410083 Hunan, China \\ Correspondence should be addressed to Dazhi Xu; xudazhi@huas.edu.cn
}

Received 4 June 2021; Revised 30 June 2021; Accepted 30 July 2021; Published 10 August 2021

Academic Editor: Mu Zhou

Copyright (c) 2021 Yan Gao and Dazhi Xu. This is an open access article distributed under the Creative Commons Attribution License, which permits unrestricted use, distribution, and reproduction in any medium, provided the original work is properly cited.

\begin{abstract}
With the development and extension of the Internet of Things technology, many fields have already involved the Internet of Things technology, and its application in dance training has gradually emerged. In order to guide dance training and solve the hidden dangers of dance training, this article mainly introduces the application of posture recognition service system based on information fusion intelligent sensor in dance training. This article uses human body detection, computer interactive Boosting algorithm calculations and establishes standard dance poses. The difference between the trainer's posture and the standard posture can be clearly seen on the posture recognition service platform. The error is only less than 5\%, and it can also help the trainer find their own mistakes in time. Dance and correct your posture in time, and it can reduce the error rate of difficult dances and reduce the error rate of $10 \%-20 \%$. In addition, through the survey on the satisfaction survey of the security services of the gesture recognition service platform, the satisfaction rate is as high as $90 \%$. Thereby, while ensuring safe training, the dance quality and level of trainers are improved.
\end{abstract}

\section{Introduction}

1.1. Research Background and Significance. The Internet of Things technology can combine the sensor personnel and terminal equipment by using local network and other communication technologies to realize the connection of people and things to form an intelligent network for remote management and control. It can provide targeted services for dance training under the condition of realizing resource sharing. Connecting dance training with the gesture recognition service platform is of great significance to the dance training process and results. In the current dance training process, the learner usually achieves the purpose of training by watching the video repeatedly or by seeking corrective guidance from the coach himself. This not only consumes a lot of time and energy but also brings certain difficulties and challenges to the trainer. Therefore, this article combines the posture recognition service platform based on information fusion smart sensors to collect standard dance moves, analyze the gap between the trainer and the standard dance, so that the trainer can intuitively feel the gap, and help the trainer discover themselves in time during dance training. By combining dance training with information fusion smart sensors to recognize postures, apply information fusion smart sensors and other technologies to capture the entire training movement and collect dance movement data and then match and compare with standard movements to analyze user movements and standards The size of the gap in the direction of the action bones allows the user to find the gap more intuitively. This method enables the majority of dance teaching workers, dancers, and dance learners who have a strong demand in teaching and self-study to make accurate adjustments to their unstandardized dance gestures, thereby ensuring the accuracy of dance gestures in training. 
At the same time, it is not restricted by time and geographical location and can be trained at any convenient time and place, which broadens the teaching methods.

1.2. Related Content. With the development of the Internet of Things technology, related research on the Internet of Things has also become an important focus. Zamanifar et al. proposed a distributed self-healing motion prediction scheme for IoT applications, namely, DSHMP-IOT, to predict the motion direction of mobile IP-based sensors in multiuser environments (such as healthcare systems) [1]. This is the first time that an AI solution has been applied to predict the direction of mobile nodes in an IP-based mobile network. The proposed scheme utilizes an implicit semi-Markov model (HSMM) to predict the direction of motion with high accuracy and low overhead. A previous work for estimating the direction of a mobile node in an IP-based mobile network was based on AOA (a hardware-specific method) [2]. Compared with similar methods, his scheme has advantages in power consumption, switching delay, and packet loss. However, there are limitations in identifying differences [3, 4]. Wilson and Bobick proposed a new method for representing, recognizing, and interpreting parametric poses [5]. The so-called parameterized posture refers to the posture that shows the spatial change of the system; the first is the point posture, where the relevant parameter is the twodimensional direction. His method is to extend the standard hidden Markov model method of gesture recognition by including global parameter changes in the output probability of the HMM state [6]. He used the visually obtained and directly measured three-dimensional body position measurement value as input. The results he gave proved the recognition advantage of PHMM over standard HMM technology and the parameter estimation compared with the noise in the input feature and higher pertinence $[7,8]$. His method is pertinent, but not universal. Chakraborty et al. found that intensive whole-body dance training may cause the fibers connecting different brain regions to spread out in a larger fan shape, increase the cross fibers, or increase the axon diameter [9]. In contrast, musicians show lower diffusion and greater fiber coherence in similar areas. Crucially, diffusion measures are related to performance in dance and music tasks, thereby distinguishing groups. This shows that dance and music training have opposite effects on the structure of WM $[10,11]$. Therefore, music training may result in a more focused focus on a specific approach to the effector. This finding emphasizes that different types of training can have different long-term effects on brain structure, thereby expanding the understanding of brain plasticity [12]. However, there is no comparative study from other aspects, and there is a certain degree of one-sidedness.

1.3. Main Content and Innovation. Through the human body detection, computer interactive calculation, and the establishment of standard dance poses, the posture recognition method based on the angle of the human joint points is improved, which can automatically collect and recognize the dance movements of the trainer. Evaluate the dance posture of the trainer in two aspects of the angle formed by the joints, and give an action comparison chart and guidance suggestions. The innovation of this paper is to use the combination of smart sensors and gesture recognition services and make full use of the advantages of smart sensors of the Internet of Things to give play to the construction and use of the gesture recognition service platform, thereby providing assistance to dance training and promoting the combined development of dance field and technology $[13,14]$.

\section{Establish a Database and Research Methods}

2.1. Boosting Algorithm. Each training set selected by the Boosting algorithm depends on the results of the previous learning. In sampling and calculation according to the error rate, it can effectively combine the errors of the last dance in dance training to achieve dance training progress [15, 16]. The Boosting algorithm can significantly reduce model prediction bias. Since the entire Boosting model is a serial combination of multiple base learners, resulting in a strong correlation between the models, the Boosting algorithm generally cannot significantly reduce the prediction variance [17]. By changing the weight distribution, the classifier can strengthen the learning of data that is defined as difficult to classify and then integrate multiple weak classifiers into a strong classifier. The algorithm deviation value is shown by

$$
\text { Bias }=E\left(\sum_{n-1}^{N} y_{n} * f_{n}\right)=y * \sum_{n-1}^{N} E\left(f_{n}\right) .
$$

Among them, Bias represents the parameter of Boosting, $E$ represents the number of dances, and $f_{n}$ represents the dancer's mastery of the dance.

\subsection{Establish a Standard Dance Movement Database. The} human body is represented as a whole that is rigidly connected by multiple joint points. Each joint point is connected by a rigid body, and each joint point represents a characteristic point, and the length of the rigid connection body is unchanged, so the human body can be. Movement is simplified to the movement of the human skeleton. In order to correct the dance moves of the trainer, there must be a standard dance move as a comparison template. In this part, by acquiring the dance moves of the dance coach, the joint points of the body are detected to form standardized motion data $[18,19]$. This article invites professional dance coaches to demonstrate standard dance moves, collects dance movement data using the gesture recognition service platform, marks each group of data with the corresponding action name, and saves it as movement information as a comparison template for trainers. The database records the standard movements of teachers of different dance styles, which can meet the training needs of beginners, and its service platform can also record new standard movements according to the different needs of trainers in the later stage.

2.3. Questionnaire Method. This article conducted a satisfaction survey on the safety of the dance studio's gesture recognition service platform. The survey respondents included dance teachers, trainers, and trainers' parents. Among them, 
there were 50 dance teachers and 100 trainers and trainers' parents. The survey questions are mainly about the satisfaction and opinions of the safety reminder service.

\section{Gesture Recognition Processing Technology and Processing Flow}

3.1. Key Points of Gesture Recognition. In dance, gesture recognition mainly relies on the recognition of joints for gesture recognition. By dividing the dancer's hand steps to be composed of nodes, using sensors to collect the key nodes of the hand, the dancer's hand data can be obtained. Through comparison, the accuracy of the dancer's hand movements can be effectively improved. The joint points of the human body are to connect the human body through the joint points. The key to joint recognition is as follows:

It can be seen from Table 1 that the key points have four parts, namely, input model diagram, extraction of body part distribution points, body joint point detection, and output of human skeleton. In this way, the dancer's posture can be more comprehensively identified and analyzed.

3.2. Gesture Recognition Processing Model. This article selects three operations of rotation, scaling and flipping. Assuming that the position of the person in the input image has been detected, this formula is calculated by recognizing the posture. The data enhancement is generated in a given detection frame [20]. Rotation includes clockwise or counterclockwise rotation around the center position. Take the first pixel in the upper left corner as the origin of the coordinates, specify the origin coordinates as $(1,1)$, rotate the original picture with the size $\left(G_{x}, G_{y}\right)$ around the center, and convert the labels of each key point into the rotated coordinate system:

$$
x_{i}^{s}=\left(x-\frac{G_{x}}{2}\right) \cos \theta-\left(y-\frac{G_{y}}{2}\right) \sin \theta+\frac{G_{x}^{s}}{2} .
$$

$x$ is the value of the coordinate system of the $i$-th key point after the rotation, the picture after the rotation is $G$, and the size is

$$
G_{x}^{s}=\left(G_{y}-G_{x} \tan \theta\right) \sin \theta+G_{x} \cos \theta .
$$

Scaling means that the image is enlarged or reduced. The image size will be larger than the original size when it is enlarged, and it needs to be cropped according to the original image size; when it is reduced, it is necessary to make assumptions about things outside the image boundary, resulting in a variety of scale transformations [21, 22]. Flip includes horizontal flip and vertical flip. It is a kind of mirror-like flip. Vertical flipping of the posture of the human body will make the human body upside down. Therefore, this article chooses horizontal flip to change the posture of the human body left and right. Suppose the coordinates $(x, y)$ of a certain key point in the original image are flipped to obtain the corresponding $j$-th key point coordinates:
TABLE 1: Key points of human joint recognition.

\begin{tabular}{lc}
\hline Serial number & Key point \\
\hline 1 & Input model diagram \\
3 & Extract the distribution points of body parts \\
4 & Body joint point detection \\
& Output human skeleton \\
\hline & $\left\{\begin{array}{l}x_{j}^{s}=G_{x}^{r}-x_{i}^{r}, \\
y_{y}^{s}=y_{s}^{r} .\end{array}\right.$
\end{tabular}

Flip in the last step to reduce the operation cost, and increase the data set while seeing the potential changes of the image; the authenticity is higher. The horizontal flip does not affect the coordinate changes of the joint points of the head, but the marking order of the corresponding joints is changed in the wrist, elbow, and other parts. By using rotation, scaling, and flipping operations on the image data, the training set of the image is enriched, so that the model has as many samples as possible, avoiding the over-fitting of a limited number of samples and improving the generalization ability of the model $[23,24]$.

3.3. Human Target Detection without Pose Restriction. Human gesture recognition can generally be divided into direct methods and indirect methods. The direct method is to establish a human body pose model, compare the collected human body data with the model one by one, and find the most similar human body pose. The indirect method is a method based on predictive generative model, which predicts the characteristic contour according to the collected human body data and recognizes the human posture according to the characteristic contour.

First, quickly find many candidate human bodies from the image to be detected. Due to the influence of noise, etc., there are many nonfalse positive human targets in the candidate body. Then, we calculate the HOG descriptors of the candidate body coverage area in the image and input the calculated HOG features into the support vector machine for classification, so that the false positive sample points of the candidate body set obtained in the first step can be removed [25]. Use the shape context to quickly detect the human target from the inside and outside of the contour image of the shape to obtain a set of points. The shape of the object in the image can be represented by these discrete points. To describe these points, they proposed a shape context descriptor. Comparing whether two points are similar can be achieved by comparing the shape context descriptors of the two points, and the similarity of the two shapes can be judged by matching the context descriptors of a discrete point set. The context descriptor of a discrete point $p_{i}$ on the shape $S$ is represented by a histogram $h_{i}$ of the relative coordinates of $p_{i}$ and other points:

$$
h_{i(k)}=\#\left\{q \neq p_{i}:\left(q-p_{i}\right) \in \operatorname{bin}(k)\right\} .
$$




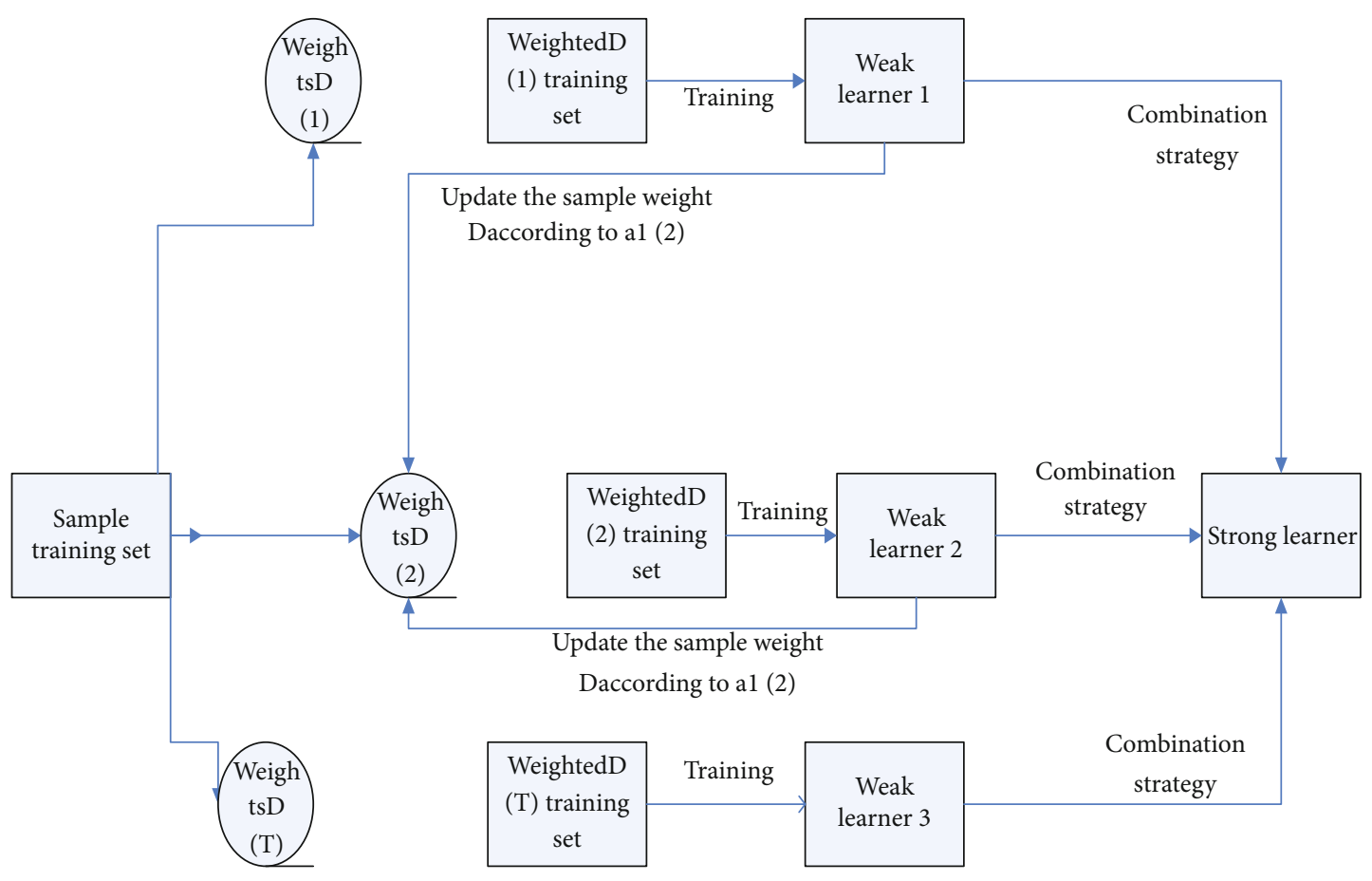

Figure 1: Boosting algorithm flow.

Next, we describe the process of applying shape context descriptor matching. We have used the human body image set to establish three human body shape templates from different perspectives [26]. For each shape template, we obtain a point set through sampling and then calculate the shape context descriptor of each point in the point set and use this set of context descriptors as the human body shape feature. Randomly sample from the image boundary to get $r$ (approximately take the set $S_{s}$ of $1 / 8$ points of the total boundary pixels). For any point $p_{i}$ of the set $S_{s}$, calculate the matching degree with any point $q j$ in the template shape $T$. The distance is defined as follows:

$$
C_{i j}^{t}=\frac{1}{2} \sum \frac{\left[h_{i}(k)-h_{j}^{t}(k)\right]^{2}}{h_{i}(k)+h_{j}^{t}(k)+\varepsilon} .
$$

In formula (2), $h_{i}$ and $h_{j}$ are the shape context descriptors of the point $p_{i}$ on the image to be detected and the point on the template image $q j$, respectively, and $h_{i}(k)$ and $h_{j}(k)$ are the $k$-th component of the descriptor. To prevent the denominator on the right side of the formula from being zero, add a small positive value to the denominator. In the scene with noisy background, the boundary obtained by applying the edge detection algorithm contains a large number of noise points [27]. In order to reduce the influence of noise, we modify the matching cost function of formula (2). In formula (2), if $h_{j}(k)$ is equal to zero and $h_{i}(k)$ is not equal to zero, we discard the difference of this component and redefine the matching distance:

$$
C_{i j}^{t}=\frac{1}{2} \sum \frac{\left[h_{i}^{\prime}(k)-h_{j}^{t}(k)\right]^{2}}{h_{i}^{\prime}(k)+h_{j}^{t}(k)+\varepsilon} .
$$

In formula (3), $h^{\prime}$ is the modified shape context descriptor, which is defined as follows:

$$
h_{i}^{\prime}(k)=\left\{\begin{array}{l}
0, \quad \text { if } h_{i}^{t}(k)=0, \\
h_{i}(k), \quad \text { else. }
\end{array}\right.
$$

The lines in the two images have similar shapes and are located at corresponding positions. The distance between the shape context descriptors should be as small as possible. The distance calculated by formula (3) is calculated by using our modified descriptor. Since the influence of noise points in the image is removed, the distance between the two descriptors is small, which is much smaller than the former. Therefore, through comparative analysis, we can effectively suppress the influence of image noise points on shape context matching by modifying the distance calculation formula. In this way, the dance form can be accurately judged.

3.4. Boosting Algorithm Flow. It can be seen from Figure 1 that the process is as follows: first, set the same weight value for each training sample, and train a weak divider under this training sample distribution. Use the weak classifier to update the weight of each sample, classify incorrectly classified samples as difficult to classify, and increase their weight, while reducing the weight of correctly classified samples to form a new weight distribution; under the new weight distribution, train a new weak classifier and update the weight 
TABLE 2: Comparison of trainer's movements and standard joint coordinates.

\begin{tabular}{lcccc}
\hline \multirow{2}{*}{ Location } & \multicolumn{2}{c}{ Standard dance joint point coordinates } & \multicolumn{2}{c}{ Trainer's joint point coordinates } \\
& Abscissa & $Y$-axis & \multicolumn{2}{c}{ Abscissa } \\
\hline Head & -50 & 100 & -55 & 95 \\
Neck & -20 & 20 & -25 & 25 \\
Left shoulder & -100 & -50 & -105 & -40 \\
Left hand & -500 & -60 & -505 & -100 \\
Right hand & 100 & -10 & 300 & 300 \\
Right shoulder & 50 & 500 & 90 & 100 \\
\hline
\end{tabular}

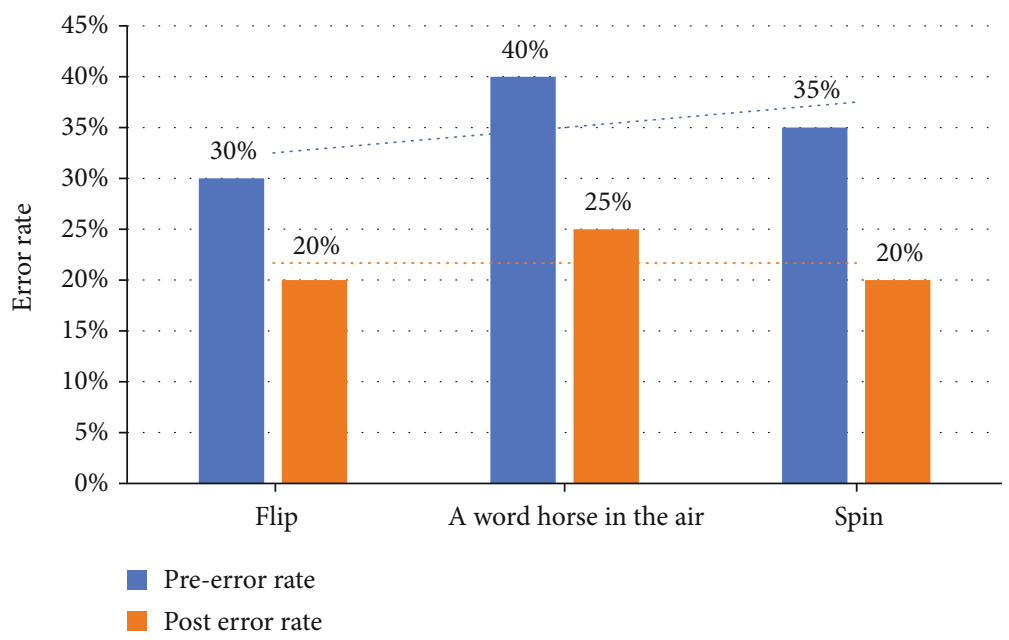

FIGURE 2: The error rate before and after the assistance of the dance recognition service platform.

value with reference to the second step. Repeat the above process $T$ times to obtain $T$ new weak classifiers. By changing the weight distribution, the classifier can strengthen learning for those data that are defined as difficult to classify. And then integrate multiple weak classifiers into a strong classifier.

\section{Dance Training Effect and Safety Satisfaction}

4.1. Dance Posture Correction Training. The correction training collects the joint coordinates of each movement of the trainer and then compares it with the standard movement of the dance instructor, as shown in the figure below. By comparing the movement trajectory of the joint points, the gap between the trainer and the standard movement can be found intuitively, and then, the learner's dance posture can be corrected.

It can be seen from Table 2 that the position coordinates of the head, neck, shoulders, and hands of the trainer and the standard dance. The dance position of the trainer's head, neck, and left shoulder is not much different from the standard dance position, and they are all within plus or minus 50 of the standard dance position. However, there is a big difference between the trainer's hand and right shoulder and the standard dance. The ordinate of the left hand differs by 50 points, and the abscissa and ordinate of the right hand differ by 200 and 290 points, respectively. At the same time, the right shoulder the ordinate also differs by 400 points. This shows that the ascending height of the trainer's arm does not meet the standard requirements, and the ascending height should be increased, and the closing movement is too fast, and it does not agree with the standard movement, resulting in a difference in the right shoulder. Therefore, trainers can follow up their own training adjustments according to the dance posture recognition service platform, perfect one's dancing posture, correct their unqualified dance postures, and reach a higher level of dance.

4.2. Strengthen the Learning of Difficult Dances. It can be seen from Figure 2 that the error rate before the assist of the flip dance is $30 \%$, the error rate after the assist is reduced to $20 \%$, the error rate of the air horse is reduced from $40 \%$ to $25 \%$, and the error rate of the rotating dance is reduced from $35 \%$. It is $20 \%$. It can be seen that the learning of difficult dance poses has been assisted by the dance recognition service platform, which reduces the difficulty and error rate of difficult dance learning, making the dancer's learning more intuitive and simple, and can easily find errors and timely correct your mistakes and show perfect dance.

4.3. Ensure the Safety of Dance Training. The dancing posture recognition service system has a calculation of the distance of the safe joint points. The safety of the dancing posture is judged by the human body in the shape context and the distance between the joint point and the joint line. When the safe distance is exceeded, the dancing posture service 


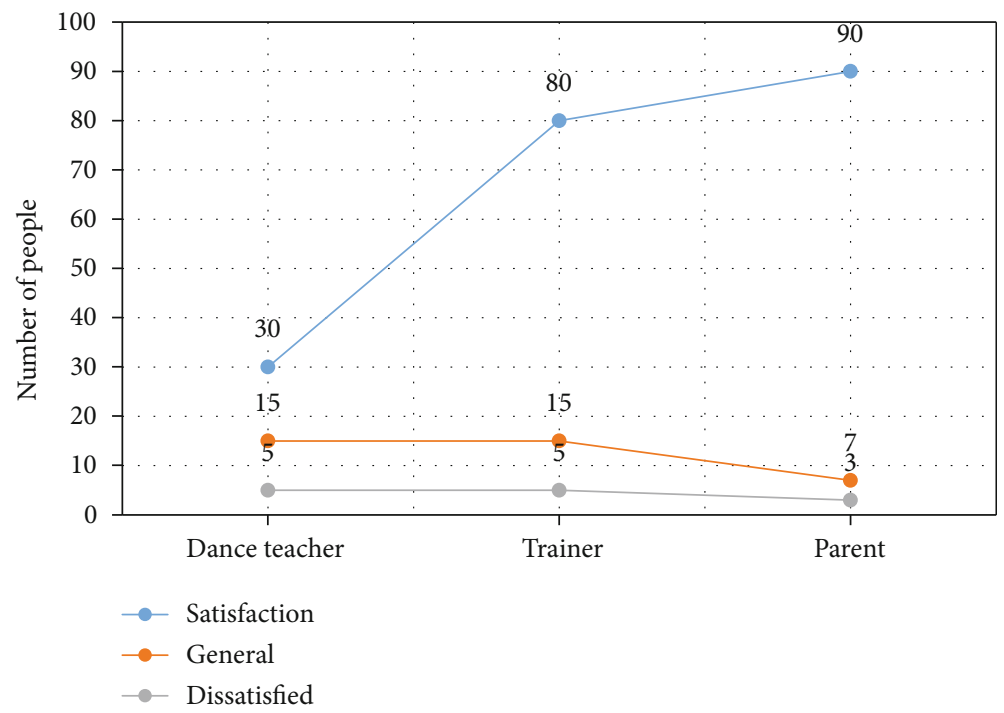

Figure 3: Service platform security service reminder satisfaction.

platform will use the camera and the connected mobile phone to perform safety reminder. The picture below is a satisfaction survey on the safety dance reminder service.

Figure 3 shows that the number of satisfied dance teachers is 30 , the number of trainers is 80 , and the number of parents of trainers is 90 . Those who feel average and dissatisfied are only a very small part of the survey population. It can be explained that the safety reminder service style in the dancing posture recognition service platform has been recognized by everyone. Especially, parents have the highest satisfaction. It also shows that the safety of dance training is an aspect that everyone attaches great importance to.

\section{Discussion}

In this paper, a dance assisted training system is designed, which can analyze and evaluate the trainer's movements from the joint point coordinates and the angle formed by the joint and provide the trainer with intuitive error correction prompts. Experiments show that the system can accurately give the difference between the trainer's movement and the standard movement, and the trainer can adjust the movement posture according to the prompts, improve the dance level, and achieve the purpose of auxiliary training. With the rapid development of technologies such as human-computer interaction, smart sensors will be widely used in various aspects such as competitive sports, rehabilitation therapy, and somatosensory games. In addition, the safe posture detection in the posture recognition service system provides real-time tripartite joint reminders, which provides a safety guarantee for the safety of dance postures in dance training. This system only collects the basic dance training movement information of some dance coaches and cannot meet the needs of a large number of dance movement training. In the future, more professional coaches' dance moves can be collected to enrich the database.

\section{Data Availability}

No data were used to support this study.

\section{Conflicts of Interest}

The authors declare that there is no conflict of interest with any financial organizations regarding the material reported in this manuscript.

\section{Acknowledgments}

This work was supported by the Project of Hunan Provincial Social Science Achievement Review Committee, China (Grant No. XSP21YBC376), and the Excellent Youth Project of Department of Education of Hunan Province, China (Grant No. 20B395).

\section{References}

[1] A. Zamanifar, E. Nazemi, and M. Vahidi-Asl, "DSHMP-IOT: a distributed self healing movement prediction scheme for internet of things applications," Applied Intelligence, vol. 46, no. 3, pp. 569-589, 2017.

[2] M. McNinch, D. Parks, R. Jacksha, and A. Miller, "Leveraging IIoT to improve machine safety in the mining industry," Mining, Metallurgy \& Exploration, vol. 36, no. 4, pp. 675-681, 2019.

[3] T. Hang, Y. Zheng, K. Qian et al., "WiSH: WiFi-based realtime human detection," Tsinghua Science and Technology, vol. 24, no. 5, pp. 615-629, 2019.

[4] M. Zhou, Y. H. Li, M. J. Tahir, X. L. Geng, Y. Wang, and W. He, "Integrated statistical test of signal distributions and access point contributions for $\mathrm{Wi}$-Fi indoor localization," IEEE Transactions on Vehicular Technology, vol. 70, no. 5, pp. 50575070, 2021.

[5] A. D. Wilson and A. F. Bobick, "Parametric hidden Markov models for gesture recognition," IEEE Transactions on Pattern 
Analysis and Machine Intelligence, vol. 21, no. 9, pp. 884-900, 1999.

[6] R. Dhall and V. K. Solanki, "An IoT based predictive connected car maintenance approach," Ijimai, vol. 4, no. 3, pp. 16-22, 2017.

[7] Z. Lu, X. Chen, Q. Li, X. Zhang, and P. Zhou, "A hand gesture recognition framework and wearable gesture-based interaction prototype for Mobile devices," IEEE Transactions on HumanMachine Systems, vol. 44, no. 2, pp. 293-299, 2014.

[8] F. Despinoy, D. Bouget, G. Forestier et al., "Unsupervised trajectory segmentation for surgical gesture recognition in robotic training," IEEE Transactions on Biomedical Engineering, vol. 63, no. 6, pp. 1280-1291, 2016.

[9] B. K. Chakraborty, D. Sarma, M. K. Bhuyan, and K. F. MacDorman, "Review of constraints on vision-based gesture recognition for human-computer interaction," IET Computer Vision, vol. 12, no. 1, pp. 3-15, 2018.

[10] M. C. Chiu and J. Y. Chiou, "Technical service platform planning based on a company's competitive advantage and future market trends: a case study of an IC foundry," Computers \& Industrial Engineering, vol. 99, pp. 503-517, 2016.

[11] D. Liu, "Research on the construction of fitness service platform in colleges and universities," International Core Journal of Engineering, vol. 6, no. 4, pp. 110-114, 2020.

[12] P. Liu, W. Jiang, X. Wang, H. Li, and H. Sun, "Research and application of artificial intelligence service platform for the power field," Global Energy Interconnection, vol. 3, no. 2, pp. 175-185, 2020.

[13] G. Plouffe and A. M. Cretu, "Static and dynamic hand gesture recognition in depth data using dynamic time warping," IEEE Transactions on Instrumentation and Measurement, vol. 65, no. 2, pp. 305-316, 2016.

[14] L. M. Sampaio, S. Subramaniam, R. Arena, and T. Bhatt, "Does virtual reality-based kinect dance training paradigm improve autonomic nervous system modulation in individuals with chronic stroke?," Journal of Vascular and Interventional Neurology, vol. 9, no. 2, pp. 21-29, 2016.

[15] L. Laberge-Côté, "The porous body: cultivating malleability in traditional dance training," Journal of Dance \& Somatic Practices, vol. 10, no. 1, pp. 65-77, 2018.

[16] F. A. Maruf, A. O. Akinpelu, B. L. Salako, and J. O. Akinyemi, "Effects of aerobic dance training on blood pressure in individuals with uncontrolled hypertension on two antihypertensive drugs: a randomized clinical trial," Journal of the American Society of Hypertension, vol. 10, no. 4, pp. 336-345, 2016.

[17] A. V. Maerle, D. V. Voronina, K. L. Dobrochaeva et al., "Immuno-PCR technology for detection of natural human antibodies against Lec disaccharide," Glycoconjugate Journal, vol. 34, no. 2, pp. 199-205, 2017.

[18] H. Ragb and V. Asari, "Multi-hypothesis approach for efficient human detection," Journal of Imaging Science and Technology, vol. 63, no. 2, pp. 20503-1-20503-13, 2019.

[19] M. Zhou, Y. M. Wang, Y. Y. Liu, and Z. S. Tian, "An information-theoretic view of WLAN localization error bound in GPS-denied environment," IEEE Transactions on Vehicular Technology., vol. 68, no. 4, pp. 4089-4093, 2019.

[20] T. Kefi-Fatteh, R. Ksantini, M. B. Kaâniche, and A. Bouhoula, "Human face detection improvement using incremental learning based on low variance directions," Signal, Image and Video Processing, vol. 13, no. 8, pp. 1503-1510, 2019.
[21] M. Man, "Kitchen contemporary dance classes in times of COVID-19, dance objects (DO): dancing the onion," Theatre, Dance and Performance Training, vol. 11, no. 4, pp. 487-487, 2020.

[22] C. Crickmay, "Drawing as part of a performance practice," Theatre, Dance and Performance Training, vol. 11, no. 4, pp. 522-523, 2020.

[23] R. Karafistan, "The five continents of theatre: facts and legends about the material culture of the actor," Theatre, Dance and Performance Training, vol. 12, no. 1, pp. 129-131, 2021.

[24] K. Beswick, "Playing to type: industry and invisible training in the National Youth Theatre's 'Playing Up 2," Theatre, Dance and Performance Training, vol. 9, no. 1, pp. 4-18, 2018.

[25] D. Kotler, M. Lynch, D. Cushman, J. Hu, and J. Garner, "Dancers' perceived and actual knowledge of anatomy," Journal of Dance Medicine \& Science, vol. 21, no. 2, pp. 76-81, 2017.

[26] C. Edinborough, "Editorial," Journal of Theatre Dance \& Performance Training, vol. 9, no. 2, pp. 139-141, 2018.

[27] B. Jennings, “Answer the question," Journal of Theatre Dance \& Performance Training, vol. 9, no. 3, pp. 430-430, 2018. 